\title{
In silico docking of urokinase plasminogen activator and integrins Bernard Degryse $^{1}$, Juan Fernandez-Recio ${ }^{2}$, Valentina Citro ${ }^{3}$, Francescol Blasi ${ }^{1}$ and Maria Vittoria Cubellis*3
}

\author{
Address: ${ }^{1}$ San Raffaele Scientific Institute, Milan, Italy, ${ }^{2}$ Life Sciences Department, Barcelona Supercomputing Center, Spain and ${ }^{3}$ Dipartimento di \\ Biologia strutturale e funzionale, Universita' di Napoli“Federico II”, Italy \\ Email: Bernard Degryse - bernard_degryse@yahoo.fr; Juan Fernandez-Recio - juanf@bsc.es; Valentina Citro - v_citro@libero.it; \\ Francescol Blasi - blasi.francesco@ hsr.it; Maria Vittoria Cubellis* - cubellis@unina.it \\ * Corresponding author
}

from Italian Society of Bioinformatics (BITS): Annual Meeting 2007

Naples, Italy. 26-28 April 2007

Published: 26 March 2008

BMC Bioinformatics 2008, 9(Suppl 2):S8 doi:10.1 I86/I47I-2105-9-S2-S8

This article is available from: http://www.biomedcentral.com/I47I-2I05/9/S2/S8

(c) 2008 Degryse et al.; licensee BioMed Central Ltd.

This is an open access article distributed under the terms of the Creative Commons Attribution License (http://creativecommons.org/licenses/by/2.0),

which permits unrestricted use, distribution, and reproduction in any medium, provided the original work is properly cited.

\begin{abstract}
Background: Urokinase, its receptor and the integrins are functionally associated and involved in regulation of cell signaling, migration, adhesion and proliferation. No structural information is available on this potential multimolecular complex. However, the tri-dimensional structure of urokinase, urokinase receptor and integrins is known.

Results: We have modeled the interaction of urokinase on two integrins, $\alpha$ llb $\beta 3$ in the open configuration and $\alpha v \beta 3$ in the closed configuration. We have found that multiple lowest energy solutions point to an interaction of the kringle domain of uPA at the boundary between $\alpha$ and $\beta$ chains on the surface of the integrins. This region is not far away from peptides that have been previously shown to have a biological role in urokinase receptor/integrins dependent signaling.

Conclusions: We demonstrated that in silico docking experiments can be successfully carried out to identify the binding mode of the kringle domain of urokinase on the scaffold of integrins in the open and closed conformation. Importantly we found that the binding mode was the same on different integrins and in both configurations. To get a molecular view of the system is a prerequisite to unravel the complex protein-protein interactions underlying urokinase/urokinase receptor/integrin mediated cell motility, adhesion and proliferation and to design rational in vitro experiments.
\end{abstract}

\section{Background}

The serine protease urokinase-type plasminogen activator (uPA) and its high affinity cell surface receptor (uPAR) play an important role in a number of physiological as well as pathological extracellular degradation processes where cell migration is required, such as fibrinolysis, inflammatory responses and tumor invasion [1]. uPA is made up of three domains, the aminoterminal growthfactor-like domain, the kringle domain and the protease domain. The first two domains form the ATF (amino terminal fragment) a domain that binds uPAR [2] and whose structure has been solved by X-ray crystallography [3]. In 
the ATF it is the growth factor domain that binds UPAR. No real function has been established so far for the kringle domain.

UPAR is a heavily glycosylated GPI-ancored protein formed by three cysteine-rich LY6-like extracellular domains (D1,D2, and D3) connected by short linker regions [4]. The three domains of UPAR are organized in a bowl-like shape with a "fissure" between domains D1 and D3 and a deep central cavity for the interaction with the growth factor domain of uPA. The whole external surface of UPAR is available for other interactions [5].

Genetic and biochemical evidence shows that uPA and uPAR are involved not only in the regulation of fibrinolysis and cell surface-focused pericellular proteolysis [2], but also in the regulation of intracellular signaling affecting cell adhesion, migration, and proliferation [1,6-9]. Some, but not all, of these functions require the proteolytic activity of uPA.

Identified interactors of $\mathrm{uPA} / \mathrm{uPAR}$ are trans-membrane signaling molecules: integrins, the $\mathrm{G}$ protein-coupled receptor FPRL1, the EGF-receptor (EGFR), the mannose-6phosphate receptor, the family of low density lipoproteins receptor-related proteins (LRP), p130 and others [1]. The involvement of integrins was originally proposed on the basis of co-immunoprecipitation experiments in hematopoietic cells [10]. Both UPA and UPAR have since been reported to interact with cell adhesion receptors of the integrin superfamily, including subfamilies $\alpha 1, \alpha 3, \alpha 5$, as well as $\alpha 2$ expressed in cells of hematopoietic lineage and containing an I (insertion) domain [11]. More recently, also the $\beta 1$ subunit has been proposed to participate in the interaction with UPAR [12].

The extracellular segments of the $\alpha$ - and $\beta$-subunits of integrins are up to 1104 and 778 residues long, respectively, with the N-terminal portions of each subunit combining to form a globular ligand-binding "head". The structure of two such integrins is known, aIIbb3 in the open configuration and $\alpha \mathrm{v} \beta 3$ in the closed configurations $[13,14]$. Integrins bind an Arg-Gly-Asp (RGD) peptide sequence, the cell recognition site present in numerous adhesive proteins. The binding site of RGD on integrin $\alpha v \beta 3$ has been identified by $x$-ray crystallography [15].

No definite information is available on the interaction of uPA or UPAR with integrins. However, three peptides were found that bound UPAR and prevented integrins function and uPAR-integrin co-immunoprecipitation. The first peptide belongs to the $\alpha$ subunit and is located in the w 4 repeat of the $\beta$-propeller. Peptide $\alpha 325$ derived from $\alpha 3 \beta 1$ integrin and $\alpha \mathrm{M} 25$ derived from $\alpha \mathrm{M} \beta 2$, were shown biochemically to directly bind uPAR, although at high con- centration, and to affect integrin and uPAR functions $[16,17]$. Also in the $\beta 1$ chain two stretches of amino acids (corresponding respectively to $\beta 1 \mathrm{P} 1$ and $\beta 1 \mathrm{P} 2$ peptides) completely inhibited UPAR-dependent cell adhesion to fibronectin, thus suggesting that they might interfere with the binding of uPAR to integrin $\alpha 5 \mathrm{~b} 1$ [12].

Despite this wealth of evidence indicating a direct interaction between UPAR and at least some integrins in vivo, evidence of a direct interaction in a purified system is lacking. Indeed, a soluble form of uPAR can be co-immunoprecipitated with purified $\alpha 3 \beta 1$ and $\alpha 5 \beta 1$ integrins, but only in the presence of uPA $[12,18]$. uPA-dependent co-immunoprecipitation was also observed in some cell lines [19]. In conclusion, even though it is absolutely clear that UPAR and integrins regulate each other, a direct interaction between UPAR and integrins is not really demonstrated and might also be (at least in certain cases) mediated by uPA.

The ligand of UPAR, UPA, regulates cell migration, adhesion and the function of $\alpha_{M} \beta 2$ integrin in cells expressing uPAR [20]. More recent evidence shows that the amino acid sequence linking the ATF to the protease domain of uPA can interact with the $\alpha \mathrm{v} \beta 3$ integrin [21].

The interaction of uPA, uPAR and integrins is important since in UPAR Ko cells at least some integrins have been shown to be inactive $[17,22]$. Thus, the identification of the mechanisms of contact between these three molecules is important. Since the 3D structure of UPA, UPAR, ATFUPAR complex, of the extracellular region of $\alpha \mathrm{v} \beta 3$ and $\alpha \operatorname{IIb} \beta 3[3,5,13,14,23,24]$ has been solved, it might be possible to exploit the available information to model these interactions. We have investigated the binding of the urokinase kringle to integrins in silico. We report that residues 113-123 of the kringle domain can be docked onto the integrin $\alpha \operatorname{IIb} \beta 3$ and $\alpha v \beta 3$ in a position that is close to regions in both the $\alpha$ and $\beta$ subunits, previously suggested to potentially interact with uPAR $[12,16]$.

\section{Results and discussion}

We docked the kringle domain of uPA on integrins in silico.

In our first experiment we docked the kringle domain onto a fragment of $\alpha \operatorname{IIb} \beta 3$ (residues 1-452 of the human $\alpha$ IIb chain, and residues $1-440$ of the human $\beta 3$ chain). This fragment represents the only possible scaffold of integrins in open conformation available in the PDB. We analysed the lowest energy solutions and in Fig.1 we arbitrarily chose to show the first 15 . Eleven out of 15 solutions clustered together contacting the integrin at the boundary between the two chains. We have highlighted on the surface of the integrin scaffold three peptides 
which were reported to play a role in integrin-uPAR and/ or integrin uPAR/ATF interaction in other integrins (no such information is available for the uPA interaction). The first peptide, which belongs to the $\alpha$ subunit and is located in the w4 repeat of the $\beta$-propeller $[16,17]$, is coloured in magenta. Its homologues, peptide $\alpha 325$ derived from $\alpha 3 \beta 1$ integrin and $\alpha \mathrm{M} 25$ derived from $\alpha \mathrm{M} \beta 2$, were shown biochemically to directly bind uPAR, although at high concentration, and to affect integrin and UPAR functions $[16,17]$. Two stretches of amino acids in the $\beta$ chain are coloured in orange and in yellow and are homologous respectively to $\beta 1 \mathrm{P} 1$ and $\beta 1 \mathrm{P} 2$ peptides derived from the $\beta$ chain of integrin $\alpha 5 \beta 1$.

A relevant biological result indicates that $\mathrm{UPA}$ is required to enhance co-immunoprecipitation of purified integrins and UPAR (see for example the paper by Degryse et al.
[18]). These data thus indicate that the kringle domain could bridge UPAR and integrins. This is compatible with the location of the cluster of low energy solutions shown in Fig.1, which localises the kringle onto the peptides homologous to $\beta 1 \mathrm{P} 1$ and $\beta 1 \mathrm{P} 2$ not distant from the peptide homologous to $\alpha 325$ which might be spanned by uPAR.

We also tested whether the same residues of the kringle domain in the complexes of the cluster were employed to contact integrin. Indeed, in 6 out 11 cases, it is the tip of the hairpin of the domain, residues 113-123 in active uPA, which contacts $\alpha 2 I I \beta 3$. These solutions ranked 2nd, 3rd, 4th, 5th, 6th and 7th when the scoring function included electrostatic energy, desolvation energy and the van der Waals term. In Fig. 2 a blow-up of the contact between the tips of four of the lowest energy solutions

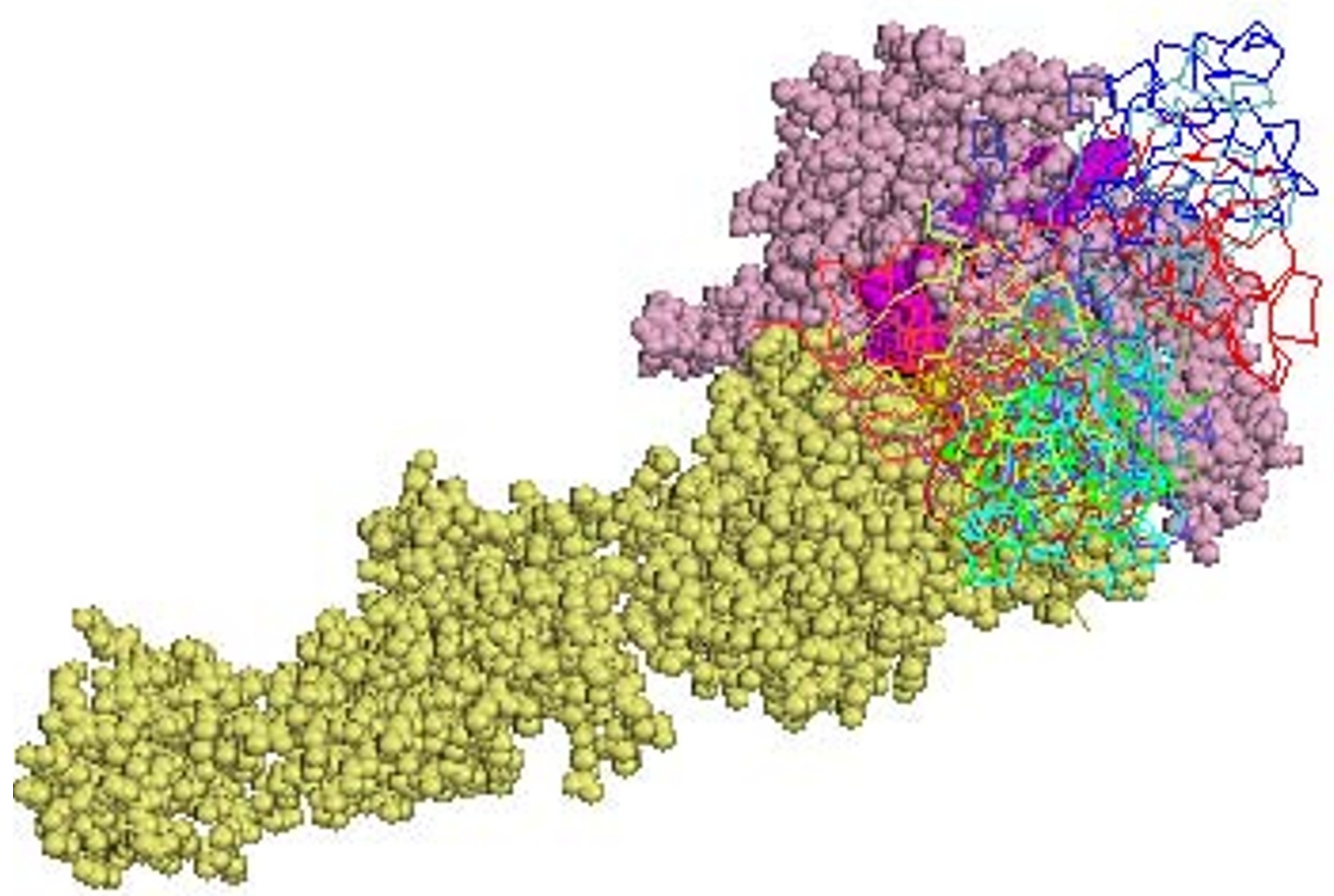

Figure I

Representation of the fifteen lowest energy poses of urokinase kringle domain on $\alpha$ llb $\beta 3$ in the open conformation. The $\alpha$ and $\beta$ chains of $\alpha$ llb $\beta 3$ are shown as pale pink and yellow spheres, respectively. The stretch of aminoacids homologous to the $\alpha 325$ peptide are highlighted in magenta, the stretch homologous to $\beta$ IPI in orange, and the one homologous to $\beta$ IP2 in yellow.

Ribbons represent every ligand positions after the rigid-body simulations. A spectrum of different shadows of red, green, blue, cyan were used going from lowest to highest energy solutions. 


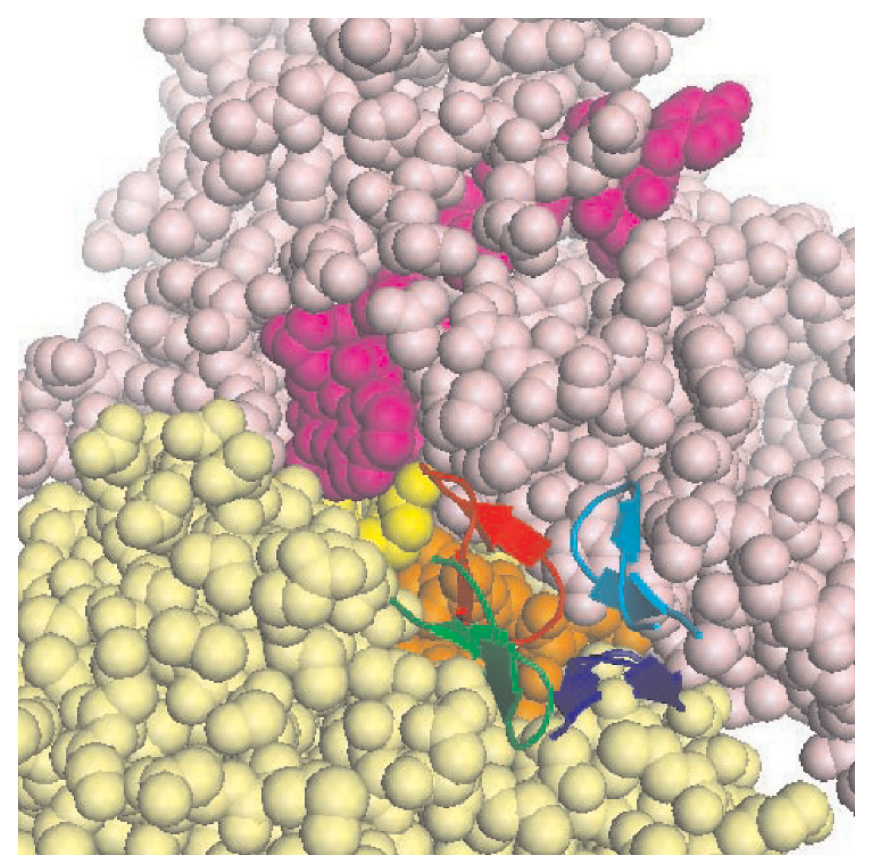

Figure 2

Kringle tip contacts with $\alpha$ llb $\beta 3$ in open conformation. The $\alpha$ and $\beta$ chains of $\alpha$ llb $\beta 3$ are shown as pale pink and yellow spheres, respectively. The amino acids stretch homologous to the $\alpha 325$ peptide is in magenta, homology to $\beta I P I$ is in orange, homology to $\beta$ IP2 in yellow. Res II -123 are shown as cartoons, those corresponding to the second ranking solution are in red, those corresponding to the third ranking solution are in green, those corresponding to the fourth ranking solution are in blue and those corresponding to the fifth ranking solution are in cyan.

(2nd, 3rd, 4th, 5th) is shown, which highlights the closeness of the kringle residues $113-123$ to both the $\alpha$ and $\beta$ subunits peptides outlined above.

We identified the residues in the integrin $\alpha$ and $\beta$ chain and on the kringle domain, which change their accessibility to solvent by more that $10 \%$ upon binding as seen in complexes ranking $2 \mathrm{nd}, 3 \mathrm{rd}, 4$ th and 5 th. These residues mostly coincide in the different solutions. In Fig.3A, we show the residues of the open configuration integrin and in Fig.3B that of the kringle domain which undergo significative shielding from solvent in all analysed complexes (2nd, 3rd, 4th and 5th). They are highlighted in blue in the $\alpha$ chain, in green in the $\beta$ chain and in red in the kringle domain. Although complexes generated by rigid body docking should be considered near native complexes because they are identified without taking into account induced fit, our results indicate that the relative orientation of the integrin and kringle domain in the low energy solutions is similar.
When we tried to dock uPAR, or uPAR in complex with growth factor domain, we did not observe a significant clustering of low energy solutions.

In uPA the catalytic serine protease moiety is preceded by a non catalytic amino-terminal fragment ATF. ATF binds the $\mathrm{uPA}$ receptor $\mathrm{uPAR}$ through its growth factor domain [2]. Visual inspection of the structure of ATF alone or in complex with uPAR reveals that the growth factor domain is a finger which fills an internal cavity of uPAR, formed by the interaction of the three domains, becoming almost completely embedded. The growth factor domain is connected by a flexible linker to the kringle, which stands as a structurally and functionally independent domain $[3,23]$. Although it is difficult to take into account the flexibility of the linker, we tried to dock the entire ATF-uPAR complex, in the conformation seen in the crystallographic structure 2i9b [3]. In nine out of ten lowest energy solutions we observed direct binding of the kringle domain to the $\alpha 2 \mathrm{II} \beta 3$ integrin (not shown).Although the solutions were quite spread, in the first and fifth ranking solutions the kringle contacted $\alpha 2 \operatorname{II} \beta 3$ at the border between $\alpha$ and $\beta$ subunits trough the tip of the hairpin of the domain, residues 113-123, i.e.as observed when docking the isolated kringle to $\alpha 2 \mathrm{II} \beta 3$ (Fig. 2).

We next carried out in silico binding of the kringle on the scaffold of an integrin in the closed configuration. The only structure available in PDB is that of $\alpha \mathrm{v} \beta 3$ ( $\alpha$ chain residues 31-987, $\beta$ chain residues, 27-718) [14]. The 15 lowest energy solutions clustered in two main groups (Fig.4). Solutions ranking 2nd, 6th, 8th, 9th, 12th, 13th and 14th when the scoring function included electrostatic energy, desolvation energy and the van der Waals term, localise in the same area already identified as a target of the kringle in the open configuration. In Fig. 5 the stretches of amino acids homologous to $\alpha 325, \beta 1 \mathrm{P} 1$ and $\beta 1 \mathrm{P} 2$ peptides are coloured in magenta, orange and yellow respectively. In this case the inclusion of the biological data and the correspondence obtained docking the kringle domain on two conformations of the integrin is required to single out correct solutions.

The same residues 113-123 of the kringle domain are likely to form the contact with the integrin in the open as well as in the closed form. This is seen in complexes ranking 8 th, 9th and 12 th. Fig. 6 shows a blow-up and the orientation of the kringle tip with respect to the stretches of amino acids homologous to $\alpha 325, \beta 1 \mathrm{P} 1$ and $\beta 1 \mathrm{P} 2$ peptides (coloured in magenta, orange and yellow). It is worth noting that complexes ranking 8th and 9th are almost superimposable. Complex ranking 12th when electrostatic energy, desolvation energy and the van der Waals term are included in the scoring function, ranks first when only electrostatic energy and desolvation energy are 

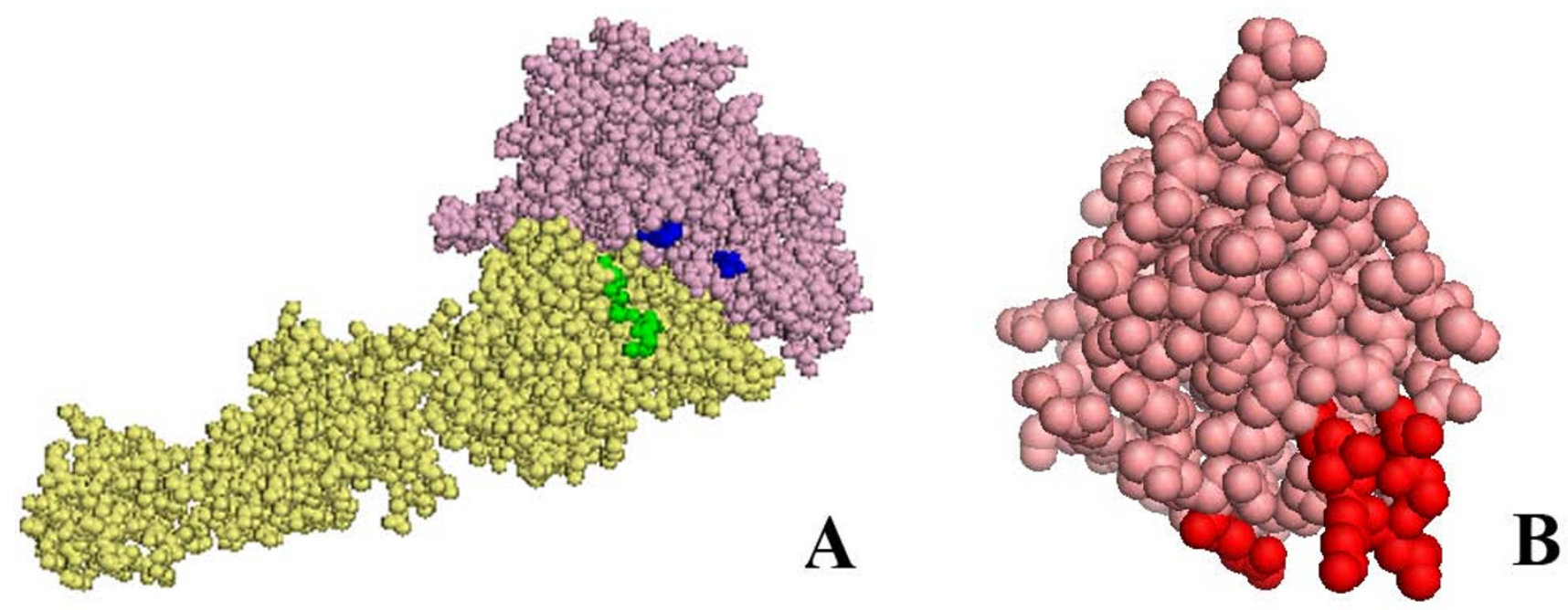

Figure 3

Residues of $\alpha \mathrm{llb} \beta 3$ in the open conformation and of urokinase kringle domain which are differentially exposed upon binding. In panel $A$ the $\alpha$ and $\beta$ chains of $\alpha$ llb $\beta 3$ are shown as pale pink and yellow spheres, respectively. Residues whose accessibility to the solvent changes by more than $10 \%$ upon kringle binding to $\alpha$ ll $\beta 3$ in the open form are highlighted in blue or green in the $\alpha$ chain or $\beta$ chain respectively.

In panel $B$ the kringle domain of urokinase is shown as salmon spheres. Residues whose accessibility to the solvent changes by more than 10\% upon binding onto $\alpha$ llb $\beta 3$ in open form are highlighted in red. In any case, only the residues differently exposed in all poses ranking second, third, fourth and fifth are highlighted.

included. It has been reported that in some cases near native docking solution are better spotted excluding van der Waals energy terms from the scoring equation because this term is too sensitive to small structural perturbations [25].

The RGD binding sequence is located very close to the stretch of aminoacids corresponding to $\beta 1 \mathrm{P} 1$ peptide and to the interaction site with the kringle domain. Thus the kringle domain might affect the binding of RGD-containing substrates to integrins. Docking solutions can be considered only representative of near native complexes and do not allow to predict whether binding of uPA to the integrin would or would not be competitive with respect to RGD.

We have identified the residues on integrin $\alpha v \beta 3 \alpha$ and $\beta$ chains and on the kringle domain which change their accessibility to solvent by more that $10 \%$ upon binding, as seen in complexes ranking 8th, 9th and 12 th. These residues mostly coincide in the different solutions. In Fig.6A we show the residues of the open configuration integrin and in Fig.6B that of the kringle domain which undergo significative shielding from solvent in all complexes analysed (8th, 9th and 12th). They are highlighted in blue in the $\alpha$ chain, in green in the $\beta$ chain and in red in the kringle domain. Comparing Fig. 3 and Fig.6, it should be noticed that the relative orientation of kringle and integrins appears to be the same in both open and close forms.

\section{Conclusions}

Our studies strongly indicate that the kringle domain can mediate the binding of uPA to integrins. The kringle domain can bind intergrin in the open and closed conforation. This interaction does not appear to be in competition with the possible direct binding of UPAR to integrins, while it might possibly interfere with the RGD-dependent binding of the integrins to its substrates. Direct molecular studies will have to address this point. However, biological studies have already indicated that the kringle is essential in the pro-adhesive effect of UPA in cells that express $\alpha_{M} \beta 2$ [20] and that truncated uPA without the uPARbinding domain can bind integrin $\alpha v \beta 3$ [21]. Our results are therefore strongly supported by these observations.

\section{Methods}

The structure of human ATF/uPAR is deposited in pdb with the code 2i9b [3]. The structure was visually inspected to derive two domains: the first domain comprises UPAR (res 1-277), growth factor like domain (res11-42 of uPA) and kringle domain (res 43-132 of uPA).

In this paper $\mathrm{uPA}$ is numbered starting from first residue after signal peptide cleaveage. 


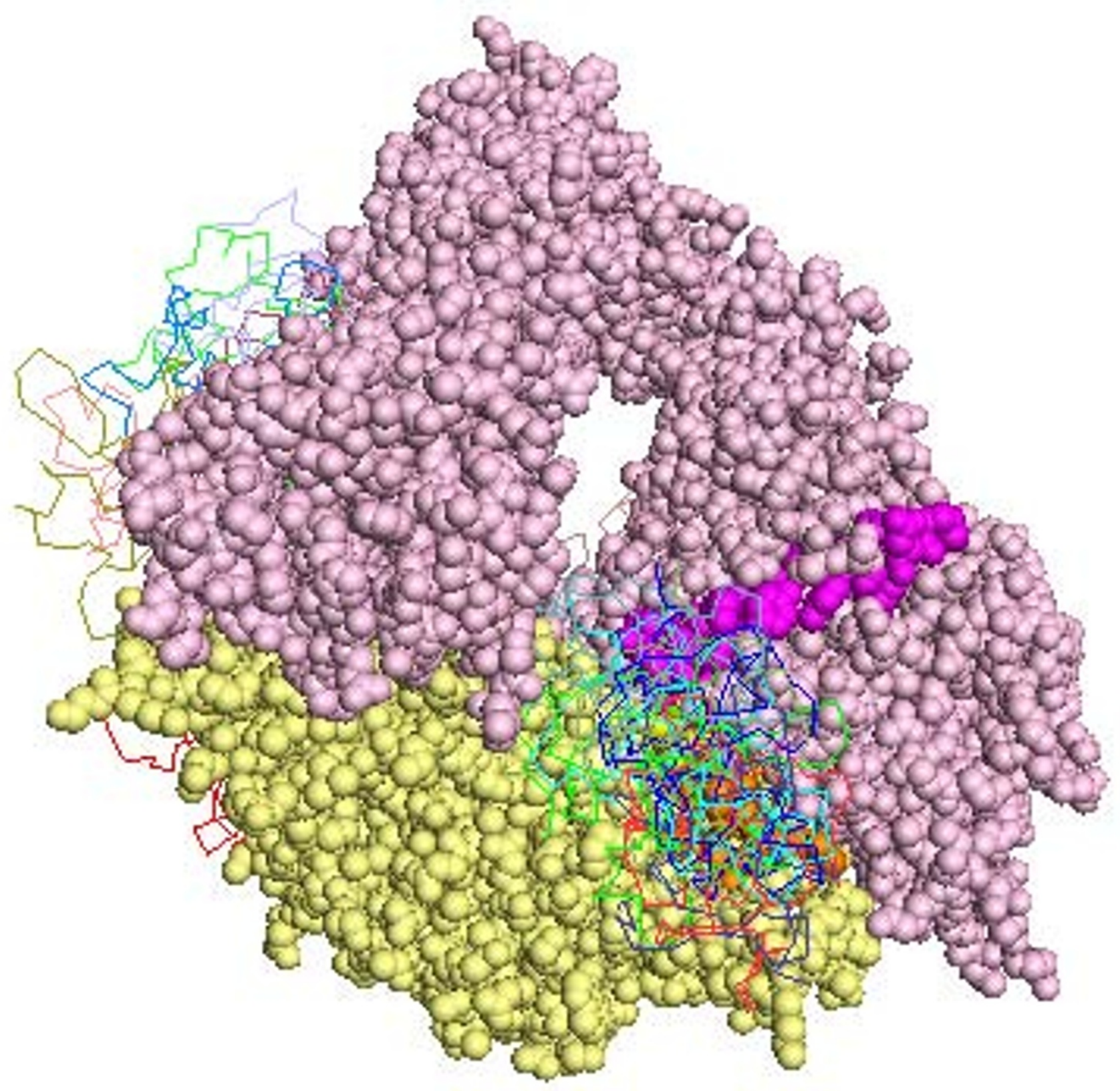

Figure 4

Representation of the fifteen lowest energy poses of urokinase kringle domain on $\alpha \mathbf{v} \beta 3$ in the closed conformation. The $\alpha$ and $\beta$ chains of $\alpha v \beta 3$ are shown as pale pink and yellow spheres respectively. The stretch of aminoacids homologous to $\alpha 325$ peptide are highlighted in magenta, homology to $\beta$ IPI is in orange, homology to $\beta$ IP2 in yellow.

Ribbons represent every ligand pose after the rigid-body simulations. A spectrum of different shadows of red, green, blue, cyan were used going from lowest to highest energy solutions.

For the integrin in open form we used the structure deposited with pdb code $1 \mathrm{jv} 2$. To solve this structure a fragment comprising residues 31-987 of the human $\alpha \mathrm{V}$ chain, and residues $27-718$ of the human $\beta 3$ chain was crystallised [14].
For the integrin in closed form we used the structure deposited with pdb code $1 \mathrm{txv}$. A fragment comprising residues 1-452 of the human $\alpha$ IIb chain, and residues 1-440 of the human $\beta 3$ chain was crystallised [13]. 


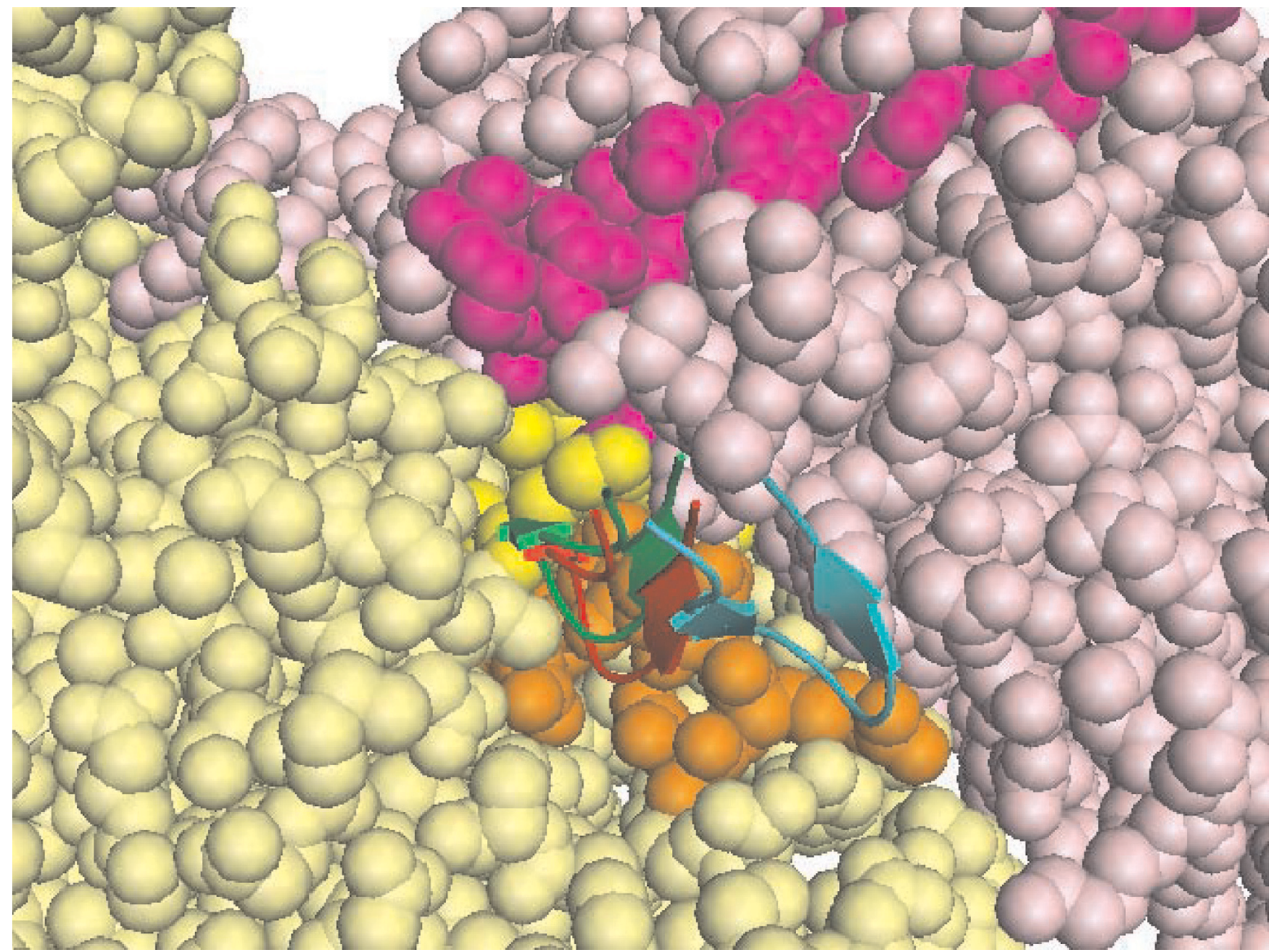

\section{Figure 5}

Kringle tip contacts with $\alpha \mathbf{v} \beta 3$ in the closed conformation. The $\alpha$ and $\beta$ chains of $\alpha v \beta 3$ are shown as pale pink and yellow spheres respectively. The stretch of amino acids homologous to the $\alpha 325$ peptide are in magenta, homology to $\beta$ IPI in orange, homology to $\beta$ IP2 in yellow. Res II3-123 are shown as cartoons, those corresponding to the eighth ranking solution are in red, those corresponding to the ninth ranking solution are in green, those corresponding to the twelfth ranking solution are in blue.

Cofactors, ions and other heteroatoms were not considered.

To build models of integrin-kringle domain comples we used the suite of docking programs called pyDock [25]. Ten thousand rigid-body docking solutions are generated by the FFT-based programs FTDOCK [26] in each experiment. Then, the docking solutions are automatically evaluated with the module pyDockSER optimised for rigidbody docking landscapes, by the equation:

$$
\text { Ebind }=\text { Eele }+ \text { Edes }+ \text { WEvdw }
$$

where Eele is the binding electrostatics energy (Coulombic potential with distance-dependent dielectric constant $\mathrm{e}=4 \mathrm{r}$, truncated to a maximum and minimum value of +1.0 and $-1.0 \mathrm{kcal} / \mathrm{mol}$, respectively) and charges from AMBER 94 force field [27]; Edes is the desolvation energy upon binding, based on atomic solvation parameters previously optimised for rigid-body docking. Evdw is the van der Waals binding energy based on the 6-12 LennardJones potential, with atomic parameters from the AMBER 94 force field, truncated to a maximum of $1.0 \mathrm{kcal} / \mathrm{mol}$ to avoid much noise from the docking of rigid body surfaces; $\mathrm{W}$ is weight which was set to 0.1 . 

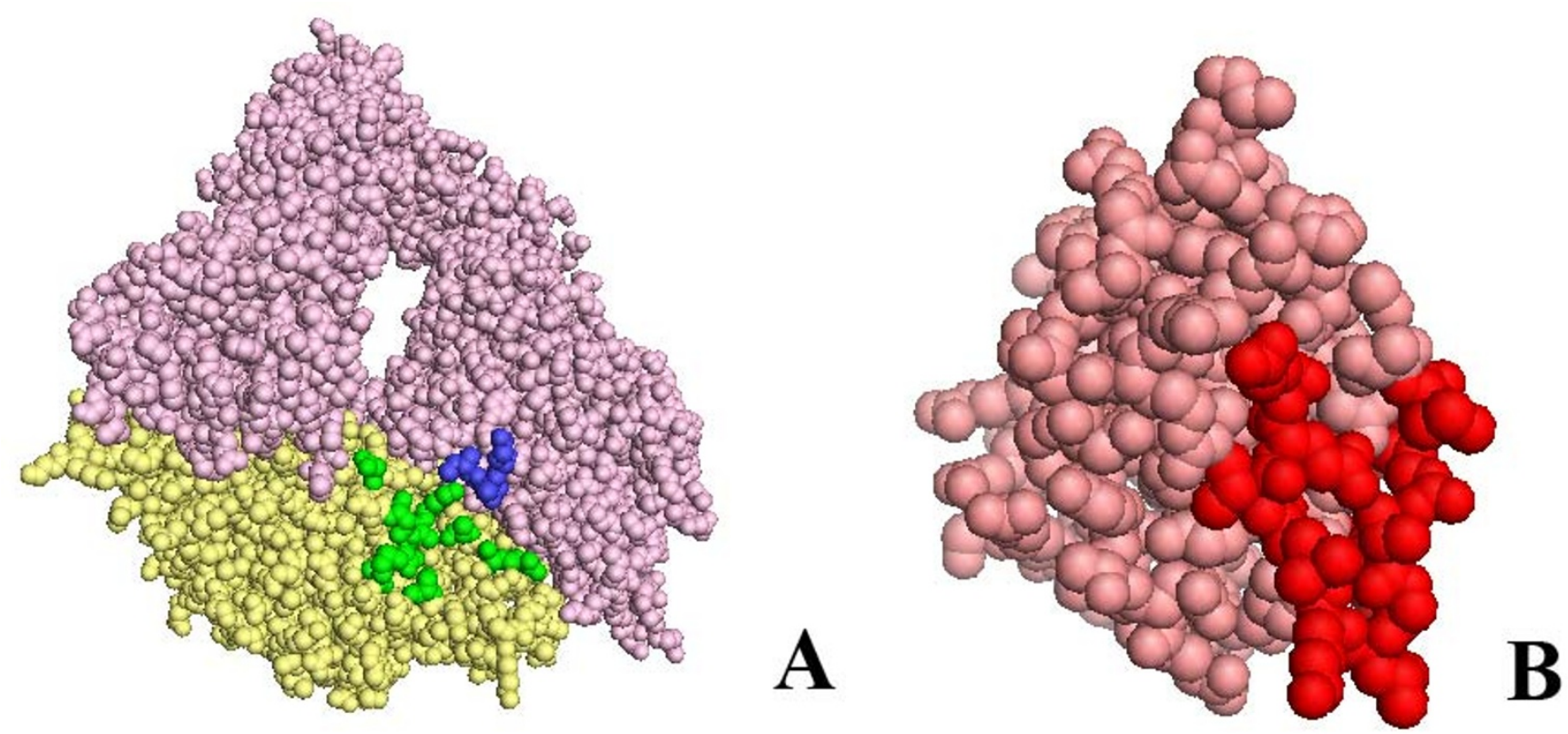

Figure 6

Residues of $\alpha v \beta 3$ in the closed conformation and of urokinase kringle domain which are differentially exposed upon binding. In panel $A$ the $\alpha$ and $\beta$ chains of $\alpha v \beta 3$ are shown as pale pink and yellow spheres respectively. Residues whose accessibility to the solvent changes by more than 10\% upon kringle binding to $\alpha v \beta 3$ in the closed conformation are highlighted in blue or green in the $\alpha$ or $\beta$ chain, respectively.

In panel $B$ the kringle domain of urokinase is shown as salmon spheres. Residues whose accessibility to the solvent changes by more than $10 \%$ upon binding onto $\alpha v \beta 3$ in the closed conformation are highlighted in red. In any case, only those residues differently exposed in the poses ranking eighth, ninth and twelfth are highlighted.

No spatial or biological restrictions were used during simulations, which allowed a complete sampling of the docking landscape around integrins

The residue solvent-accessible area were calculated using Naccess [28] with a $1.4 \AA$ probe radius

Figures were drawn with Pymol [29].

\section{List of abbreviations used}

uPA: urokinase type plasminogen activator

ATF: amino terminal fragment of the urokinase type plasminogen activator

UPAR: urokinase type plasminogen activator receptor

EGF: epidermal growth factor

FPRL1: formyl peptide receptor-like 1

LRP: low density lipoproteins receptor

GPI: Glycosylphosphatidylinisotol

\section{Competing interests}

All the authors declare that they have no competing interests.

\section{Authors' contributions}

MVC conceived the study and designed the experiments. JFR provided coding and discussion on the methodology. MVC carried out the experiments. MVC and FB wrote the manuscript. VC helped in the elaboration of data. BDG provided information about the biological system.

\section{Acknowledgements}

This work was supported by grants from MIUR PRIN 2005 (to M.V.C.) and by the program for short mobility of Federico II Naples University (to M.V.C).

This article has been published as part of BMC Bioinformatics Volume 9 Supplement 2, 2008: Italian Society of Bioinformatics (BITS): Annual Meeting 2007. The full contents of the supplement are available online at http:// www.biomedcentral.com/I47/-2/05/9? issue $=$ S2

\section{References}

I. Blasi F, Carmeliet P: uPAR: a versatile signalling orchestrator. Nat Rev Mol Cell Biol 2002, 3:932-43.

2. Appella E, Robinson EA, Ullrich SJ, Stoppelli MP, Corti A, Cassani G, Blasi F: The receptor-binding sequence of urokinase. A biological function for the growth-factor module of proteases. J Biol Chem 1987, 262:4437-40. 
3. Barinka C, Parry G, Callahan J, Shaw DE, Kuo A, Bdeir K, Cines DB, Mazar A, Lubkowski J: Structural basis of interaction between urokinase-type plasminogen activator and its receptor. J Mol Biol 2006, 363:482-95.

4. Ploug M, Ellis V: Structure-function relationships in the receptor for urokinase-type plasminogen activator. Comparison to other members of the Ly-6 family and snake venom alphaneurotoxins. FEBS Lett 1994, 349:163-8.

5. Llinas P, Le Du MH, Gardsvoll H, Dano K, Ploug M, Gilquin B, Stura EA, Menez A: Crystal structure of the human urokinase plasminogen activator receptor bound to an antagonist peptide. Embo J 2005, 24:1655-63.

6. Chapman HA: Plasminogen activators, integrins, and the coordinated regulation of cell adhesion and migration. Curr Opin Cell Biol 1997, 9:7|4-24.

7. Ossowski L, Aguirre-Ghiso JA: Urokinase receptor and integrin partnership: coordination of signaling for cell adhesion, migration and growth. Curr Opin Cell Biol 2000, I 2:6|3-20.

8. Preissner KT, Kanse SM, May AE: Urokinase receptor: a molecular organizer in cellular communication. Curr Opin Cell Biol $2000,12: 621-8$

9. Kjoller L: The urokinase plasminogen activator receptor in the regulation of the actin cytoskeleton and cell motility. Biol Chem 2002, 383:5-19.

10. Bohuslav J, Horejsi V, Hansmann C, Stockl J, Weidle UH, Majdic O, Bartke I, Knapp W, Stockinger H: Urokinase plasminogen activator receptor, beta 2-integrins, and Src-kinases within a single receptor complex of human monocytes. J Exp Med 1995, | 81: | 381 -90.

II. Wei Y, Eble JA, Wang Z, Kreidberg JA, Chapman HA: Urokinase receptors promote betal integrin function through interactions with integrin alpha3beta I. Mol Biol Cell 200I, I 2:2975-86.

12. Wei Y, Czekay RP, Robillard L, Kugler MC, Zhang F, Kim KK, Xiong JP, Humphries MJ, Chapman HA: Regulation of alpha5betal integrin conformation and function by urokinase receptor binding. J Cell Biol 2005, I68:50I-II.

13. Xiao T, Takagi J, Coller BS, Wang JH, Springer TA: Structural basis for allostery in integrins and binding to fibrinogen-mimetic therapeutics. Nature 2004, 432:59-67.

14. Xiong JP, Stehle T, Diefenbach B, Zhang R, Dunker R, Scott DL, Joachimiak A, Goodman SL, Arnaout MA: Crystal structure of the extracellular segment of integrin alpha Vbeta3. Science 200I, 294:339-45.

15. Xiong JP, Stehle T, Zhang R, Joachimiak A, Frech M, Goodman SL, Arnaout MA: Crystal structure of the extracellular segment of integrin alpha Vbeta3 in complex with an Arg-Gly-Asp ligand. Science 2002, 296: $15 \mid-5$.

16. Wei Y, Lukashev M, Simon DI, Bodary SC, Rosenberg S, Doyle MV, Chapman HA: Regulation of integrin function by the urokinase receptor. Science 1996, 273:155|-5.

17. Simon DI, Wei Y, Zhang L, Rao NK, Xu H, Chen Z, Liu Q, Rosenberg $S$, Chapman HA: Identification of a urokinase receptor-integrin interaction site. Promiscuous regulator of integrin function. J Biol Chem 2000, 275: 10228-34.

18. Degryse B, Resnati M, Czekay RP, Loskutoff DJ, Blasi F: Domain 2 of the urokinase receptor contains an integrin-interacting epitope with intrinsic signaling activity: generation of a new integrin inhibitor. J Biol Chem 2005, 280:24792-803.

19. Mazzieri R, D'Alessio S, Kenmoe RK, Ossowski L, Blasi F: An uncleavable uPAR mutant allows dissection of signaling pathways in uPA-dependent cell migration. Mol Biol Cell 2006, I 7:367-78.

20. Pluskota E, Soloviev DA, Plow EF: Convergence of the adhesive and fibrinolytic systems: recognition of urokinase by integrin alpha Mbeta 2 as well as by the urokinase receptor regulates cell adhesion and migration. Blood 2003, I 01:1582-90.

21. Franco P, Vocca I, Carriero MV, Alfano D, Cito L, Longanesi-Cattani I, Grieco P, Ossowski L, Stoppelli MP: Activation of urokinase receptor by a novel interaction between the connecting peptide region of urokinase and alpha $\mathbf{v}$ beta 5 integrin. J Cell Sci 2006, I 19:3424-34.

22. May AE, Kanse SM, Lund LR, Gisler RH, Imhof BA, Preissner KT: Urokinase receptor (CD87) regulates leukocyte recruitment via beta 2 integrins in vivo. J Exp Med 1998, 188: 1029-37.

23. Huang M, Mazar AP, Parry G, Higazi AA, Kuo A, Cines DB: Crystallization of soluble urokinase receptor (suPAR) in complex with urokinase amino-terminal fragment ( I- | 43). Acta Crystallogr D Biol Crystallogr 2005, 6 I:697-700.

24. Li Y, Parry G, Chen L, Callahan JA, Shaw DE, Meehan EJ, Mazar AP, Huang M: An anti-urokinase plasminogen activator receptor (uPAR) antibody: crystal structure and binding epitope. J Mol Biol 2007, 365: I I 17-29.

25. Cheng T, Blundell T.L., Fernandez-Recio J: Proteins. pyDock: electrostatics and desolvation for effective scoring of rigid-body protein-protein docking 2007.

26. Gabb HA, Jackson RM, Sternberg MJ: Modelling protein docking using shape complementarity, electrostatics and biochemical information. J Mol Biol 1997, 272:106-20.

27. CP Cornell WD, Bayly Cl, Gould IR, Merz KM, Ferguson DM, Spellmayer DC, Fox T, Cadwell JW, Kollman PA: A second generation force field for the simulation of protein, nucleic acidis, and organic molecules. J Am Chem Soc 1995, I 17:5179-5197.

28. TJ Hubbard SJ: NACCESS. In Department of Biochemistry and Molecular Biology University College London; 1993.

29. DeLano WL: The PyMOL Molecular Graphics System on World Wide Web. 2002. http://www.pymol.org.

Publish with Bio Med Central and every scientist can read your work free of charge

"BioMed Central will be the most significant development for disseminating the results of biomedical research in our lifetime. "

Sir Paul Nurse, Cancer Research UK

Your research papers will be:

- available free of charge to the entire biomedical community

- peer reviewed and published immediately upon acceptance

- cited in PubMed and archived on PubMed Central

- yours - you keep the copyright
BioMedcentral 